\title{
SINGULARITY FORMATION FOR A FLUID MECHANICS MODEL WITH NONLOCAL VELOCITY
}

\author{
CHANGHUI TAN
}

\begin{abstract}
We study a 1D fluid mechanics model with nonlocal velocity. The equation can be viewed as a fractional porous medium flow, a 1D model of quasi-geostrophic equation, and also a special case of the Euler-Alignment system. For strictly positive smooth initial data, global regularity has been proved in 11. We construct a family of non-negative smooth initial data so that solution loses $C^{1}$ regularity. Our result indicates that strict positivity is a critical condition to ensure global regularity of the system. We also extend our construction to the corresponding models in multi-dimensions.
\end{abstract}

\section{INTRODUCTION}

We are interested in the following $1 \mathrm{D}$ continuity equation

$$
\partial_{t} \rho+\partial_{x}(\rho u)=0
$$

with a nonlocal velocity field

$$
u=H \Lambda^{\alpha-1} \rho, \quad 0<\alpha<2,
$$

where $H$ is the Hilbert transform, and $\Lambda^{s}=(-\Delta)^{s / 2}$ denotes the nonlocal fractional Laplacian operator. The initial density is set to be non-negative

$$
\left.\rho(x, t)\right|_{t=0}=\rho_{0}(x) \geq 0 .
$$

The dynamics of $\rho$ in the system (1)-(3) can be alternatively written as

$$
\partial_{t} \rho+u \partial_{x} \rho=-\rho \Lambda^{\alpha} \rho .
$$

It consists a nonlocal transport term $u \partial_{x} \rho$, and a dissipation term $-\rho \Lambda^{\alpha} \rho$ which is nonlinear and nonlocal.

Without the dissipation term, the equation is an active scaler

$$
\partial_{t} \rho+u \partial_{x} \rho=0
$$

with the velocity $u$ defined in (2). It arises as $1 \mathrm{D}$ simplified models for $2 \mathrm{D}$ surface quasigeostrophic equations. For $\alpha=1$, equation (5) was studied by Córdoba, Córdoba and Fontelos [9], where a finite time loss of $C^{1}$ regularity is shown for some initial data. Silvestre and Vicol [17] proved the similar behavior for $\alpha \in(0,2)$. Both results indicate that the transport term intends to drive the dynamics into singularity in finite time.

Date: February 13, 2019.

2010 Mathematics Subject Classification. 35Q35, 35Q92.

Key words and phrases. porous medium flow, quasi-geostrophic equations, Euler-Alignment system, singularity. 
With the dissipation term, the equation (4) appears in many models in fluid mechanics. Since the dissipation term has a possible regularizing effect, the understanding of the competition between the transport term and the dissipation term attracts a lot of attentions in recent years.

Fractional porous medium flow. The main system (11)-(3) can be viewed as a porous medium equation with fractional potential pressure, where $\rho$ represents the density of the fluid. It was introduced by Caffarelli and Vázquez [3, where an existence theory for weak solutions was established, for $\rho_{0} \in L^{1}$. The regularizing effect was discussed in a series of successive works: 2] for $\alpha \in(0,1) \cup(1,2)$, and [4] for $\alpha=1$. Their result states that weak solutions of the system with any $L^{1}$ initial data instantly becomes Hölder continuous, and stays in $C^{\gamma}$ for all time, with some $\gamma \in(0,1)$. Such regularizing effect is proved in higher dimensions as well.

For $\alpha=1$, Carrillo, Ferreira and Precioso [6] studied the system in the space of probability measures with bounded second moment. They established a global wellposedness theory by taking advantage of the gradient flow structure of the system in 1D.

The system is also related to a model for the motion of the dislocations in a solid proposed by Biler, Karch and Monneau in [1].

1D model of quasi-geostrophic equation. Chae, Córdoba, Córdoba and Fontelos 8 considered (11)-(3) with $\alpha=1$. They interpreted the system as a 1D simplified model of 2D quasi-geostrophic equation in atmospheric science, where $\rho$ represents the temperature of the air subject to a shift ( $\rho=\theta+\kappa$ in their notations).

They studied the system in the periodic domain $\mathbb{T}=[-1 / 2,1 / 2]$, and focused on propagation of regularity with smooth initial data. The result consists two parts. First, they showed that if $\rho_{0}>0$, then all $H^{3}$ initial data stays in $H^{3}$ in all time. Second, they proved that the system loses $C^{1}$ regularity in finite time, with the initial data chosen as

$$
\rho_{0}(x)=1-\cos (2 \pi x), \quad x \in \mathbb{T} .
$$

The main difference between the two types of initial data is that $\rho_{0}(x)=0$ is attained in the latter case. It indicates that the preservation of $C^{1}$ regularity critically depends on the strict positivity of the initial data.

In [7, Castro and Córdoba discussed the blowup phenomenon for more general initial data without strict positivity.

It is worth noting that $u=H \rho$ when $\alpha=1$. Some properties and identities of Hilbert transform were crucially used in their proof. So, the extension of the result to general $\alpha \in(0,2)$ is far from trivial.

Euler-Alignment system. System (1)-(3) is also related to a biologically motivated complex interacting system modeling collective behaviors. The Cucker-Smale model [10] is an agent-based model governed by Newton's second law

$$
\dot{x}_{i}=v_{i}, \quad m \dot{v}_{i}=F_{i}:=\frac{1}{N} \sum_{j=1}^{N} \psi\left(\left|x_{i}-x_{j}\right|\right)\left(v_{j}-v_{i}\right),
$$

where $\left(x_{i}, v_{i}\right)_{i=1}^{N}$ represent the position and velocity of agent $i$. The force $F_{i}$ describes the alignment interaction on velocity, where the influence function $\psi$ characterizes the strength of the velocity alignment between two agents. Natually, it is a decreasing function of the distance between the agents. 
The macroscopic representation of Cucker-Smale model (7), derived through a kinetic system (see [12]), is called Euler-Alignment system. In 1D, it reads

$$
\begin{aligned}
\partial_{t} \rho+\partial_{x}(\rho u) & =0, \\
\partial_{t} u+u \partial_{x} u & =\int_{\mathbb{R}} \psi(|x-y|)(u(y, t)-u(x, t)) \rho(y, t) d y .
\end{aligned}
$$

For the case when $\psi$ is Lipschitz, the system was studied in [18, 5]. A critical threshold phenomenon was discovered: preservation of $C^{1}$ regularity depends on the choice of initial data. Subcritical initial data lead to global regularity, while supercritical initial data lead to fintie time shock formation.

Another case is when $\psi$ is singular, taking the form

$$
\psi(|x|)=\frac{c_{\alpha}}{|x|^{1+\alpha}}, \quad 0<\alpha<2,
$$

with $c_{\alpha}$ be a positive constant such that

$$
\Lambda^{\alpha} f=c_{\alpha} \int_{\mathbb{R}} \frac{f(x)-f(y)}{|x-y|^{1+\alpha}} d y .
$$

One interesting feature of such choice of $\psi$ is that, equation (9) becomes closely related to the Burgers equation with fractional dissipation

$$
\partial_{t} u+u \partial_{x} u=-\Lambda^{\alpha} u,
$$

by enforcing $\rho \equiv 1$. Kiselev, Nazarov and Shterenberg [13] studied (11): when $0<\alpha<1$, there exists initial data leading to finite time blow up; when $\alpha \in[1,2)$, all smooth initial data lead to global regularity.

The Euler-Alignment system (8)-(9) with singular influence function (10) was studied in [1] in the periodic domain. It was shown that all smooth initial data $\rho_{0}>0$ leads to global regularity. In particular, in the range of $\alpha \in(0,1)$, the behavior of the solution is very different from the Burgers equation with fractional dissipation, despite their similarity. The global regularity result is extended to more general singular influence function in [14]. Moreover, it is shown that the $C^{1}$ norm of the density $\rho$ is uniformly bounded in all time. For $\alpha \in[1,2)$, global regularity was independently shown by Shvydkoy and Tadmor in [15] through a different approach. Their result can also be extended for $\alpha \in(0,1)$ in [16].

As discussed in [11], a useful reformulation of the Euler-Alignment system for $\rho$ and $G=\partial_{x} u-\Lambda^{\alpha} \rho$ has the form

$$
\partial_{t} \rho+\partial_{x}(\rho u)=0, \quad \partial_{t} G+\partial_{x}(G u)=0, \quad \partial_{x} u=\Lambda^{\alpha} \rho+G .
$$

In particular, if we pick the initial data such that $G_{0}(x)=\partial_{x} u_{0}(x)-\Lambda^{\alpha} \rho_{0}(x) \equiv 0$, then $G \equiv 0$ for all $t>0$, and the dynamics of $\rho$ becomes our main system (11)-(2).

Therefore, the result in [11] implies that for $\alpha \in(0,2)$, system (11)-(2) with smooth initial data $\rho_{0}>0$ stays smooth in all time. It serves as an extension to the first part of the result in [8] with general $\alpha$.

The main result. In this paper, we focus on (1)-(2) with non-negative initial data $\rho_{0}$ which is not strictly positive. We construct initial data which lead to singularity formations.

Theorem 1.1. Consider the system (11)-(3) in the periodic domain $\mathbb{T}$. There exists a family of smooth initial data $\rho_{0}$ such that the solution $\rho(\cdot, t)$ is not bounded in $C^{1}$ uniformly in $t$. 
Theorem 1.1 says that the solution will lose $C^{1}$ regularity as time approaches infinity. Note that this type of singularity does not happen when $\rho_{0}>0$ (see [14]). Hence, the non-vacuum assumption is critical to ensure global regularity.

Theorem 1.1 extends the blow up result in [8] to the general case $\alpha \in(0,2)$. However, it only guarantees singularity formations as time approaches infinity. Whether the blowup happens in finite time is still an open problem, which requires future investigations.

As a direct consequence, we have the following result for Euler-Alignment system.

Corollary 1.2. Consider the initial value problem of Euler-Alignment system (8)-(9) with singular influence function $\psi$ defined in (10). There exists smooth initial data $\rho_{0} \geq 0$ and $u_{0}$ such that the solution lose uniform $C^{1}$ regularity.

The choice of initial data could be $\rho_{0}$ from Theorem 1.1, and $u_{0}=H \Lambda^{\alpha-1} \rho_{0}$.

The rest of the paper is organized as follows. In section 2, we show apriori bounds for the system with some proposed symmetry. In section 3, we obtain an enhanced estimate on the velocity $u$, which plays an essential rule in proving the singularity formation. Theorem 1.1 is then proved in section 4. In section 5, we extend the result to systems in multidimensional spaces. Finally, in section 6, we make some remarks on related topics for further investigation.

\section{Apriori estimates}

In this section, we derive some useful estimates for our main system (11)-(3), which will help us to construct initial data and obtain finite time blow up.

We first propose the following even symmetry condition to $\rho_{0}$

$$
\rho_{0}(x)=\rho_{0}(-x) \text {. }
$$

Since we consider periodic data, $\rho_{0}$ can be determined by its value in $x \in[0,1 / 2]$. We also note that periodicity and even symmetry preserves in time.

2.1. Maximum principle. Let us assume the initial data is bounded, satisfying

$$
0 \leq \rho_{0}(x) \leq \bar{\rho}, \quad \forall x \in \mathbb{T} .
$$

Then, $\rho(\cdot, t)$ satisfies ( $\mathrm{H2}$ ) for all $t \geq 0$, due to maximum principle.

Proposition 2.1 (Maximum principle). Let $\rho$ be a smooth solution of (1) with initial data

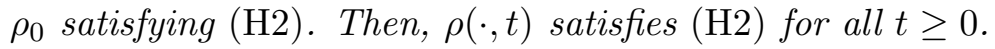

Proof. Suppose $\rho(x, t) \leq \bar{\rho}$ does not hold for all $(x, t)$. Then, there exists $x_{0}$ and $t_{0}$ such that

$$
\rho\left(x_{0}, t_{0}\right)=\bar{\rho}, \quad \rho\left(x, t_{0}\right) \leq \bar{\rho}, \forall x \in \mathbb{T}, \quad \text { and } \quad \partial_{t} \rho\left(x_{0}, t_{0}\right)>0 .
$$

So the violation first occurs at $x_{0}$ at time $t_{0}+$.

Since $\rho\left(\cdot, t_{0}\right)$ attains its maximum at $x_{0}$, we know

$$
\partial_{x} \rho\left(x_{0}, t_{0}\right)=0, \quad \text { and } \quad \Lambda^{\alpha} \rho\left(x_{0}, t_{0}\right) \geq 0 .
$$

Therefore, from (4) we obtain

$$
\partial_{t} \rho\left(x_{0}, t_{0}\right)=-u\left(x_{0}, t_{0}\right) \partial_{x} \rho\left(x_{0}, t_{0}\right)-\rho\left(x_{0}, t_{0}\right) \Lambda^{\alpha} \rho\left(x_{0}, t_{0}\right) \leq 0 .
$$

This leads to a contradiction. Therefore, $\rho(x, t) \leq \bar{\rho}$ holds for all $x \in \mathbb{T}$ and $t \geq 0$.

Positivity preserving property $\rho(x, t) \geq 0$ can be proved similarly. 
2.2. Conservation of mass. We denote $m$ as the initial mass

$$
m=\int_{\mathbb{T}} \rho_{0}(x) d x .
$$

Integrating the continuity equation (11) in $x$, we get

$$
\frac{d}{d t} \int_{\mathbb{T}} \rho(x, t) d x=-\int_{\mathbb{T}} \partial_{x}(\rho(x, t) u(x, t))=0 .
$$

This implies the conservation of total mass.

Moreover, the mass in any interval is conserved along the characteristic flow.

Proposition 2.2 (Conservation of mass). Let $\rho$ be a strong solution of the continuity equation (1). Let $X_{1}(t), X_{2}(t)$ be two characteristic paths starting at $x_{1}$ and $x_{2}$, respectively.

$$
\frac{d}{d t} X_{i}(t)=u\left(X_{i}(t), t\right), \quad X_{i}(0)=x_{i}, \quad i=1,2 .
$$

Then, the mass in the interval $\left[X_{1}(t), X_{2}(t)\right]$ is conserved in time, namely

$$
\int_{X\left(t ; x_{1}\right)}^{X\left(t ; x_{2}\right)} \rho(x, t) d x=\int_{x_{1}}^{x_{2}} \rho_{0}(x) d x, \quad \forall t \geq 0 .
$$

The proof can be found, for instance, in [19, Lemma 5.1].

2.3. Preservation of monotonicity. We make another assumption on $\rho_{0}$.

$$
\rho_{0}(0)=0, \quad \partial_{x} \rho_{0}(x) \geq 0, \forall x \in[0,1 / 2],
$$

namely $\rho_{0}$ is increasing in $[0,1 / 2]$.

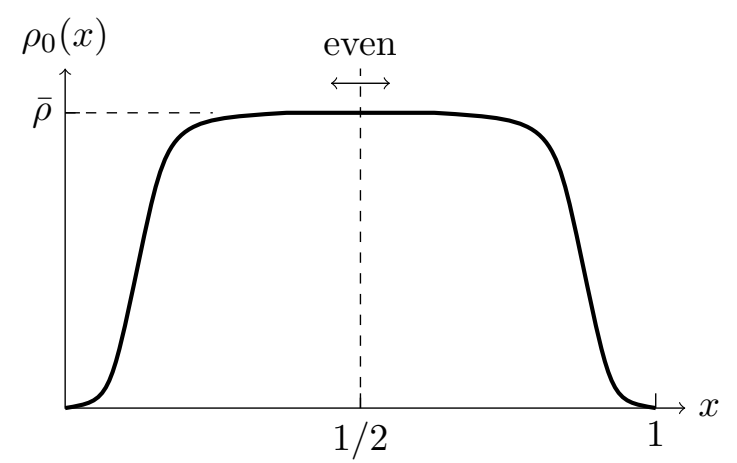

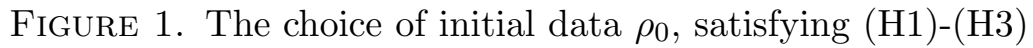

The following proposition shows that such monotonicity is preserved in time.

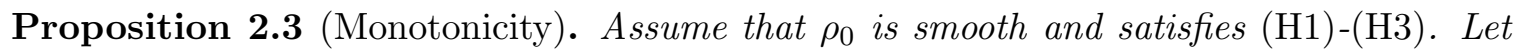
$\rho$ be a classical solution of (11)-(3). Then, $\rho(\cdot, t)$ satisfies (픈 for any $t \geq 0$.

Proof. Let us denote $\zeta:=\partial_{x} \rho$, and write down its dynamics by differentiating (4) in $x$

$$
\partial_{t} \zeta=-u \partial_{x} \zeta-2 \zeta \partial_{x} u-\rho \partial_{x}^{2} u=-u \partial_{x} \zeta-2 \zeta \Lambda^{\alpha} \rho-\rho \Lambda^{\alpha} \zeta
$$

By periodicity and ( $(\mathrm{H1})$, we know $\zeta(\cdot, t)$ is odd, and so

$$
\zeta(0, t)=\zeta(1 / 2, t)=0 .
$$


Our goal is to prove $\zeta(x, t) \geq 0$, for all $x \in[0,1 / 2]$ and $t \geq 0$. Assume the argument is false, then there exist at time $t_{0}$ and position $x_{0} \in(0,1 / 2)$ such that the solution satisfies

$$
\zeta\left(x_{0}, t_{0}\right)=0, \quad \zeta\left(x, t_{0}\right) \geq 0, \forall x \in[0,1 / 2], \quad \text { and } \quad \partial_{t} \zeta\left(x_{0}, t_{0}\right)<0,
$$

so that the break down first happens at $\left(x_{0}, t_{0}+\right)$.

Since $\zeta\left(\cdot, t_{0}\right)$ reaches a local minimum at $x_{0}$, clearly $\partial_{x} \zeta\left(x_{0}, t_{0}\right)=0$. Therefore, the dynamics (13) at $\left(x_{0}, t_{0}\right)$ becomes

$$
\partial_{t} \zeta\left(x_{0}, t\right)=-\rho\left(x_{0}, t_{0}\right) \Lambda^{\alpha} \zeta\left(x_{0}, t_{0}\right) .
$$

From Proposition 2.1, we know $\rho\left(x_{0}, t_{0}\right) \geq 0$. So, we are left to estimate $\Lambda^{\alpha} \zeta\left(x_{0}, t_{0}\right)$.

$$
\begin{aligned}
\Lambda^{\alpha} \zeta\left(x_{0}, t_{0}\right) & =c_{\alpha} \int_{\mathbb{R}} \frac{\zeta\left(x_{0}, t_{0}\right)-\zeta\left(y, t_{0}\right)}{\left|x_{0}-y\right|^{1+\alpha}} d y=-c_{\alpha} \sum_{l \in \mathbb{Z}} \int_{-1 / 2}^{1 / 2} \frac{\zeta\left(y, t_{0}\right)}{\left|x_{0}-y-l\right|^{1+\alpha}} d y \\
& =-c_{\alpha}\left[\sum_{l \in \mathbb{Z}} \int_{0}^{1 / 2} \frac{\zeta\left(-y, t_{0}\right)}{\left|x_{0}+y-l\right|^{1+\alpha}} d y+\sum_{l \in \mathbb{Z}} \int_{0}^{1 / 2} \frac{\zeta\left(y, t_{0}\right)}{\left|x_{0}-y-l\right|^{1+\alpha}} d y\right] \\
& =-c_{\alpha} \int_{0}^{1 / 2} \zeta\left(y, t_{0}\right) \sum_{l \in \mathbb{Z}}\left(\frac{1}{\left|x_{0}-y-l\right|^{1+\alpha}}-\frac{1}{\left|x_{0}+y-l\right|^{1+\alpha}}\right) d y .
\end{aligned}
$$

From (14) and the following Lemma 2.4, we conclude that $\Lambda^{\alpha} \zeta\left(x_{0}, t_{0}\right) \leq 0$ and hence $\partial_{t} \zeta\left(x_{0}, t_{0}\right) \geq 0$. This contradicts with the last inequality in (14).

Lemma 2.4. Suppose $x, y \in[0,1 / 2]$ and $\alpha>0$. Then

$$
\sum_{l \in \mathbb{Z}}\left(\frac{1}{|x-y-l|^{1+\alpha}}-\frac{1}{|x+y-l|^{1+\alpha}}\right) \geq 0
$$

Proof. We first consider the case when $y \leq x$. The sum can be rewritten as

$$
\sum_{l \geq 1}\left[\left(\frac{1}{(l-1+x-y)^{1+\alpha}}-\frac{1}{(l-x-y)^{1+\alpha}}\right)-\left(\frac{1}{(l-1+x+y)^{1+\alpha}}-\frac{1}{(l-x+y)^{1+\alpha}}\right)\right] .
$$

Define

$$
H_{l}(z)=\frac{1}{(l-1+x-z)^{1+\alpha}}-\frac{1}{(l-x-z)^{1+\alpha}} .
$$

Then, the sum can be represented as

$$
\sum_{l \geq 1}\left(H_{l}(y)-H_{l}(-y)\right)
$$

Since we have

$$
H_{l}^{\prime}(z)=(1+\alpha)\left[\frac{1}{(l-1+x-z)^{2+\alpha}}-\frac{1}{(l-x-z)^{2+\alpha}}\right] \geq 0, \quad \forall z \in[-1 / 2,1 / 2],
$$

we get $H_{l}(y)-H_{l}(-y) \geq 0$ for any $y \in[0, x]$. It implies that the sum is non-negative.

The case when $y>x$ can be treated in the same way. 
2.4. An estimate on velocity. The velocity $u$ defined in (2) can be expressed in the integral form as follows:

$$
u(x, t)=c_{\alpha} \int_{\mathbb{R}} \frac{\rho(y, t)-\rho(x, t)}{\operatorname{sgn}(x-y)|x-y|^{\alpha}} d y .
$$

Fix $x \in[0,1 / 2]$ and $t \geq 0$. We decompose the integrand and use (푸) to get

$$
\begin{aligned}
\frac{1}{c_{\alpha}} u(x, t)= & \int_{0}^{\infty} \frac{\rho(y, t)-\rho(x, t)}{|x+y|^{\alpha}} d y+\int_{0}^{x} \frac{\rho(y, t)-\rho(x, t)}{|x-y|^{\alpha}} d y-\int_{x}^{\infty} \frac{\rho(y, t)-\rho(x, t)}{|x-y|^{\alpha}} d y \\
= & \int_{0}^{x}(\rho(y, t)-\rho(x, t))\left(\frac{1}{(x+y)^{\alpha}}+\frac{1}{(x-y)^{\alpha}}\right) d y \\
& +\int_{x}^{\infty}(\rho(y, t)-\rho(x, t))\left(\frac{1}{(x+y)^{\alpha}}-\frac{1}{(y-x)^{\alpha}}\right) d y=: I+I I .
\end{aligned}
$$

Due to monotonicity condition of $\rho(\cdot, t)(\underline{\mathrm{H} 3})$, we know that the first term $I \leq 0$. For the second term $I I$, observe that

$$
\frac{1}{(x+y)^{\alpha}}-\frac{1}{(y-x)^{\alpha}}<0, \quad \forall y>x>0 .
$$

So, the integral in $I I$ can be decompose into two parts:

$$
\int_{x}^{\infty}=\sum_{l=0}^{\infty} \int_{l+x}^{l+1-x}+\sum_{l=1}^{\infty} \int_{l-x}^{l+x}
$$

Again, condition ( $(\mathrm{H} 3)$ implies that for the first part $\rho(y, t)-\rho(x, t) \geq 0$, and for the second part $\rho(y, t)-\rho(x, t) \leq 0$. Let us denote $I I=I I_{1}+I I_{2}$ where $I I_{1}$ and $I I_{2}$ represents the corresponding integrals. Then, $I I_{1} \leq 0$ and $I I_{2} \geq 0$.

The next lemma shows $I+I I_{2} \leq 0$, at least when $x$ is sufficiently small.

Lemma 2.5. There exists a $\delta=\delta(\alpha)>0$, such that for all $x \in[0, \delta], I+I I_{2} \leq 0$.

Proof. Let us first write

$$
I I_{2}=\int_{-x}^{x}(\rho(x, t)-\rho(y, t)) \sum_{l=1}^{\infty}\left(\frac{1}{(y+l-x)^{\alpha}}-\frac{1}{(y+l+x)^{\alpha}}\right) d y .
$$

Using mean value theorem, we have for $y \in(-x, x)$,

$$
\frac{1}{(y+l-x)^{\alpha}}-\frac{1}{(y+l+x)^{\alpha}} \leq \alpha(l-2 x)^{-1-\alpha} \cdot(2 x) .
$$

Therefore,

$$
\sum_{l=1}^{\infty} \frac{1}{(y+l-x)^{\alpha}}-\frac{1}{(y+l+x)^{\alpha}} \leq 2 \alpha x\left[(1-2 x)^{-1-\alpha}+\int_{1}^{\infty}(z-2 x)^{-1-\alpha} d z\right] \leq C x .
$$

For $x \leq 1 / 4$, the last inequality holds with the choice of $C=2^{\alpha+1}(1+2 \alpha)$.

Now, let us put together $I$ and $I I_{2}$.

$$
\begin{aligned}
I+I I_{2}= & \int_{0}^{x}(\rho(x, t)-\rho(y, t))\left[-\frac{1}{(x+y)^{\alpha}}-\frac{1}{(x-y)^{\alpha}}\right. \\
& \left.+\sum_{l=1}^{\infty}\left(\frac{1}{(y+l-x)^{\alpha}}-\frac{1}{(y+l+x)^{\alpha}}+\frac{1}{(-y+l-x)^{\alpha}}-\frac{1}{(-y+l+x)^{\alpha}}\right)\right] d y
\end{aligned}
$$




$$
\begin{aligned}
& \leq \int_{0}^{x}(\rho(x, t)-\rho(y, t))\left[-\frac{1}{(x-y)^{\alpha}}+0+2 C x\right] d y \\
& \leq\left(-x^{-\alpha}+2 C x\right) \int_{0}^{x}(\rho(x, t)-\rho(y, t)) d y .
\end{aligned}
$$

We pick a small enough $\delta$ as follows

$$
\delta=\min \left\{\frac{1}{4},\left(\frac{1}{3 C}\right)^{\frac{1}{1+\alpha}}\right\},
$$

Then, for any $x \in(0, \delta]$, we have $-x^{-\alpha}+2 C x \leq-C x<0$.

Also, the monotonicity condition ( $\mathrm{H} 3 \mathrm{l})$ implies that

$$
\int_{0}^{x}(\rho(x, t)-\rho(y, t)) d y \geq 0 .
$$

Therefore, conclude that $I+I I_{2} \leq 0$ for all $x \in[0, \delta]$.

Lemma 2.5 directly implies the following estimate on $u$.

Theorem 2.6. Let $\rho$ be a classical solution of (11)-(3), with periodic initial data $\rho_{0}$ satisfying (1ㅡ) $-(\mathrm{H} 3)$. Let $\delta$ be defined as (16). Then, the velocity

$$
u(x, t) \leq 0, \quad \forall x \in[0, \delta], t \geq 0 .
$$

One may remove the smallness assumption on $x$ in Theorem 2.6 by a more careful estimate on $I_{2}$. For our purpose, it is enough to consider small $x$.

\section{An ENHANCED EStimate ON Velocity} Recall

In order to show singularity formations, we need a stronger estimate on the velocity.

$$
u(x, t)=\left(I+I I_{2}\right)+I I_{1} .
$$

Lemma 2.5 ensures $I+I I_{2} \leq 0$. The estimate $I I_{1} \leq 0$ simply follows for (플 $)$.

We aim to improve our estimate on

$$
I I_{1}=-\sum_{l=0}^{\infty} \int_{l+x}^{l+1-x}(\rho(y, t)-\rho(x, t))\left(\frac{1}{(y-x)^{\alpha}}-\frac{1}{(y+x)^{\alpha}}\right) d y .
$$

An easy observation is that, if $\rho(x, t)=\bar{\rho}$, then $I I_{1}=0$. In this case, it is not possible to get any improvement. Therefore, we obtain an enhanced estimate when $\rho(x, t)$ is small.

Theorem 3.1. Let $\rho$ be a classical solution of (11)-(13), with periodic initial data $\rho_{0}$ satisfying

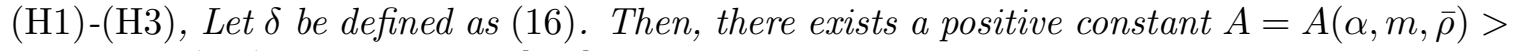
0 , for any $(x, t)$ satisfying $x \in[0, \delta]$ and

$$
\rho(x, t) \leq \frac{m}{2},
$$

the velocity

$$
u(x, t) \leq-A x .
$$

Let us explain the main idea of the proof. We focus on a better bound on $[x, 1 / 2]$, and use the rough bound by zero for the rest of the integrand.

$$
I I_{1} \leq-\int_{x}^{1 / 2}(\rho(y, t)-\rho(x, t))\left(\frac{1}{(y-x)^{\alpha}}-\frac{1}{(y+x)^{\alpha}}\right) d y .
$$


Denote the term that we concern by $I I I$.

$$
I I I=\int_{x}^{1 / 2}(\rho(y, t)-\rho(x, t)) h(x, y) d y, \quad h(x, y)=\frac{1}{(y-x)^{\alpha}}-\frac{1}{(y+x)^{\alpha}} .
$$

To obtain a lower bound on $I I I$, we need several observations. First, for a fixed $x \in[0, \delta]$, $h(x, y) \geq 0$ for any $y \in(x, 1 / 2]$. Moreover,

$$
\partial_{y} h(x, y)=-\alpha\left[\frac{1}{(y-x)^{\alpha+1}}-\frac{1}{(y+x)^{\alpha+1}}\right] \leq 0 .
$$

Next, we apply (플 $(\underline{\mathrm{H} 3})$, and get

$$
0 \stackrel{\text { (H33) }}{\leq} \rho(y, t)-\rho(x, t) \stackrel{\sqrt{\mathrm{H} 2} 2)}{\leq} \bar{\rho}-\rho(x, t), \quad \forall y \in(x, 1 / 2] .
$$

Moreover, the assumption (17) implies

$$
\int_{x}^{1 / 2}(\rho(y, t)-\rho(x, t)) d y \stackrel{\sqrt{\mathrm{H} 3})}{\geq} \int_{0}^{1 / 2}(\rho(y, t)-\rho(x, t)) d y=\frac{m}{2}-\frac{\rho(x, t)}{2} \stackrel{\sqrt{17})}{\geq} \frac{m}{4} .
$$

The following lemma is helpful to get a positive lower bound of III.

Lemma 3.2. Let $f$ be a positive decreasing function on $[a, b] . \lambda$ and $M$ are positive constant such that $\lambda<M(b-a)$. Then,

$$
\min _{\omega}\left\{\int_{a}^{b} \omega(x) f(x) d x \mid 0 \leq \omega(x) \leq M, \int_{a}^{b} \omega(x) d x \geq \lambda\right\}=M \int_{b-\frac{\lambda}{M}}^{b} f(x) d x .
$$

The minimum is attained at

$$
\omega_{\min }(x)=\left\{\begin{array}{ll}
0 & a \leq x<b-\frac{\lambda}{M} \\
M & b-\frac{\lambda}{M} \leq x \leq b
\end{array} .\right.
$$

Proof. First, it is easy to check $\omega_{\min }$ satisfies

$$
0 \leq \omega(x) \leq M, \quad \int_{a}^{b} \omega(x) d x \geq \lambda .
$$

We will prove that for any $\omega$ which satisfies (22), $\int_{a}^{b}\left(\omega(x)-\omega_{\min }(x)\right) f(x) d x \geq 0$. Compute

$$
\int_{a}^{b}\left(\omega(x)-\omega_{\min }(x)\right) f(x) d x=\int_{a}^{b-\frac{\lambda}{M}} \omega(x) f(x) d x+\int_{b-\frac{\lambda}{M}}^{b}(\omega(x)-M) f(x) d x .
$$

From the first condition in (22), we know $\omega(x) \geq 0$ and $\omega(x)-M \leq 0$. Together with the assumption that $f$ is positive and decreasing, we obtain

$$
\begin{aligned}
\int_{a}^{b}\left(\omega(x)-\omega_{\min }(x)\right) f(x) d x & \geq f\left(b-\frac{\lambda}{M}\right) \int_{a}^{b-\frac{\lambda}{M}} \omega(x) d x+f\left(b-\frac{\lambda}{M}\right) \int_{b-\frac{\lambda}{M}}^{b}(\omega(x)-M) d x \\
& =f\left(b-\frac{\lambda}{M}\right)\left[\int_{a}^{b} \omega(x) d x-M \cdot \frac{\lambda}{M}\right] \geq f\left(b-\frac{\lambda}{M}\right)(\lambda-\lambda)=0 .
\end{aligned}
$$

Hence, we conclude

$$
\min _{\omega \text { satisfies (22) }} \int_{a}^{b} \omega(x) f(x) d x=\int_{a}^{b} \omega_{\min }(x) f(x) d x=M \int_{b-\frac{\lambda}{M}}^{b} f(x) d x .
$$


Putting together (19), (20) and (21), we can apply Lemma 3.2 with

$$
f(y)=h(x, y), \omega(y)=\rho(y, t)-\rho(x, t), \lambda=\frac{m}{4}, M=1-\rho(x, t), a=x, b=\frac{1}{2} .
$$

Then,

$$
I I I \geq(1-\rho(x, t)) \int_{\frac{1}{2}-\frac{m}{4(\bar{\rho}-\rho(x, t))}}^{\frac{1}{2}} h(x, y) d y \geq(1-\rho(x, t)) \int_{\frac{1}{2}-\frac{m}{4 \bar{\rho}}}^{\frac{1}{2}} h(x, y) d y .
$$

Using the mean value theorem, we have

$$
h(x, y) \geq \frac{\alpha}{(y+x)^{1+\alpha}} \cdot(2 x) \geq 2 \alpha x .
$$

Finally, we obtain

$$
I I I \geq \frac{1}{2} \cdot \frac{m}{4 \bar{\rho}} \cdot(2 \alpha x)=\frac{\alpha m}{4 \bar{\rho}} x,
$$

and therefore

$$
I I_{1} \leq-\frac{\alpha m}{4 \bar{\rho}} x
$$

We end up with the improved estimate (18) with $A=\frac{\alpha m}{4 \bar{\rho}}$.

\section{Singularity FOrmation}

In this section, we prove Theorem 1.1: for any smooth initial data satisfying (ㅍ1)-( the solution loses uniform $C^{1}$ regularity.

We will argue by contradiction. Suppose the solution is uniformly $C^{1}$ in all time, then there exists $\varepsilon>0$ such that

$$
u(\varepsilon, t) \leq \frac{m}{2}, \quad \forall t \geq 0 .
$$

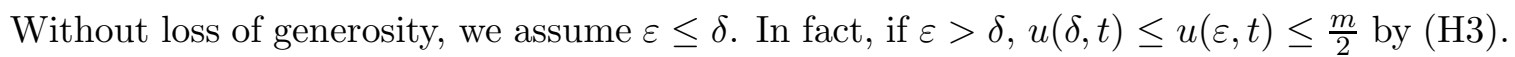
We can then take $\varepsilon=\delta$.

Let us denote $X(t ; x)$ be the characteristic path initiated at $x$, satisfying

$$
\frac{d}{d t} X(t ; x)=u(X(t ; x), t), \quad X(0 ; x)=x .
$$

By symmetry, we know $u(0, t)=0$ and hence $X(t ; 0)=0$ for all $t \geq 0$.

Define $m(x, t)$ be the mass in the interval $[0, x]$ at time $t$ :

$$
m(x, t):=\int_{0}^{x} \rho(x, t) d x .
$$

We apply Proposition 2.2 and get

$$
m(X(t ; x), t)=m(x, 0) .
$$

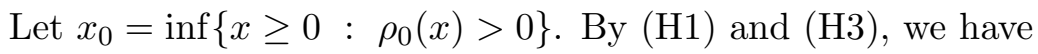

$$
\operatorname{supp}\left(\rho_{0}\right)=\left(x_{0}, 1-x_{0}\right) .
$$

We shall proceed with two cases. 
Case 1: $x_{0}<\varepsilon$. By the definition of $x_{0}$, we know $\rho_{0}(\varepsilon)>0$. Moreover, $m(\varepsilon, 0)>0$.

By Theorem 2.6. we know $X(t ; \varepsilon) \leq \varepsilon$ for any $t \geq 0$. Then, the assumption (23) ensures that $\rho(X(t ; \varepsilon)) \leq \frac{m}{2}$ in all time. This allows us to use the enhanced estimate, Theorem 3.1 , and get

$$
u(X(t ; \varepsilon), t) \leq-A X(t ; \varepsilon),
$$

where $A>0$ does not depend on $\varepsilon$ or $t$.

Then, we can integrate along the characteristic path, and get

$$
X(t ; \varepsilon) \leq \varepsilon e^{-A t} .
$$

A simple estimate yields

$$
m(X(t ; \varepsilon), t)=\int_{0}^{X(t ; \varepsilon)} \rho(x, t) d x \stackrel{\sqrt{\mathrm{H} 3})}{\leq} X(t ; \varepsilon) \rho(X(t ; \varepsilon), t) \leq \frac{\varepsilon m}{2} e^{-A t} .
$$

This contradicts with the mass conservation (24) if we pick $t$ large enough, more precisely,

$$
t>\frac{1}{A} \log \frac{\varepsilon m}{2 m(\varepsilon, 0)} .
$$

Remark 4.1. If $\rho_{0}(x)=0$ only at a single point $x=0$, then $x_{0}=0$. No matter what $\varepsilon$ is, we are always under this case. Therefore, we have already shown the singularity formation. Note that the initial data (6) lie into this category.

Case 2: $x_{0} \geq \varepsilon$. If $x_{0}>0$, namely $\rho_{0}(x)=0$ in an interval $\left[-x_{0}, x_{0}\right]$, it is possible that $\varepsilon \leq x_{0}$. Then, $m(\varepsilon, 0)=0$. Consequently, the right hand side of (25) is not bounded any more.

To obtain a contradiction, we first examine the characteristic path starting at $x_{0}$. Since $\rho_{0}\left(x_{0}\right)=0$, it is easy to see that $\rho_{0}\left(X\left(t ; x_{0}\right), t\right)=0$ at any time. We can apply the enhanced estimate (18) at $\left(X\left(t ; x_{0}\right), t\right)$, and obtain

$$
X\left(t ; x_{0}\right) \leq x_{0} e^{-A t} .
$$

Then, there exists a finite time $T_{*}$ such that $X\left(t ; x_{0}\right) \leq \varepsilon$. For instance, one can take

$$
T_{*}=\frac{1}{A} \log \frac{x_{0}}{\varepsilon} .
$$

Now, we consider the characteristic path that goes through the point $\left(\varepsilon, T_{*}+1\right)$. If the flow is smooth, we can track back and find a unique point $x_{*}$ such that $\varepsilon=X\left(T_{*}+1 ; x_{*}\right)$.

Moreover, as $X\left(T_{*}+1 ; x_{0}\right)<\varepsilon$, we have $x_{0}<x_{*}$. By the definition of $x_{0}$, we know $\rho_{0}\left(x_{*}\right)>0$ and hence $m\left(x_{*}, 0\right)>0$.

Now, we can repeat the argument in case 1 along $X\left(t ; x_{*}\right)$. First, apply the enhanced estimate (18) at $\left(X\left(t ; x_{*}\right), t\right)$ for $t \geq T_{*}+1$ and get

$$
X\left(t ; x_{*}\right) \leq \varepsilon e^{-A\left(t-\left(T_{*}+1\right)\right)}, \quad \forall t \geq T_{*}+1 .
$$

Next, we estimate the mass

$$
m\left(X\left(t ; x_{*}\right), t\right) \leq X\left(t ; x_{*}\right) \rho\left(X\left(t ; x_{*}\right), t\right) \leq \frac{\varepsilon m}{2} e^{-A\left(t-\left(T_{*}+1\right)\right)}, \quad \forall t \geq T_{*}+1 .
$$

Finally, take $t$ large enough

$$
t>\frac{1}{A} \log \frac{\varepsilon m}{2 m\left(x_{*}, 0\right)}+\left(T_{*}+1\right) .
$$

Then, $m\left(X\left(t ; x_{*}\right), t\right)<m\left(x_{*}, 0\right)$, which contradicts with the mass conservation (24). 


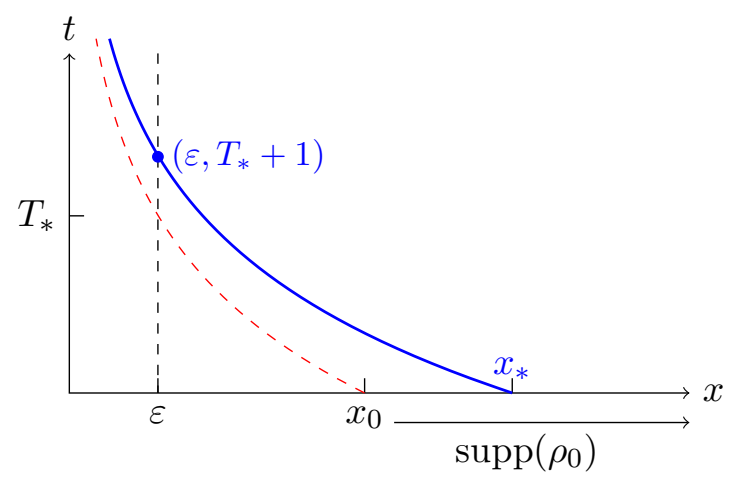

Figure 2. The characteristic path that leads to a contradiction

\section{EXTENSION TO SYSTEMS IN MULTI-DIMENSIONS}

In this section, we extend our main result to systems in higher dimensions. The main idea is to consider $\rho_{0}(\mathbf{x})=\rho_{0}\left(x_{1}\right)$ and reduce the system to $1 \mathrm{D}$ so that our construction can be used.

5.1. Fractional porous medium flow. Let us recall the fractional porous medium flow in multi-dimension

$$
\partial_{t} \rho+\nabla \cdot(\rho \mathbf{u})=0, \quad \mathbf{u}=\nabla \Lambda^{\alpha-2} \rho,
$$

with $\mathbf{x}=\left(x_{1}, \cdots, x_{n}\right) \in \mathbb{T}^{n}$ and $0<\alpha<2$.

Fix any time $t$ and drop the time dependence for simplicity. Assume $\rho(\mathbf{x})=\rho\left(x_{1}\right)$, namely $\rho$ is a constant in $\left(x_{2}, \cdots, x_{n}\right)$ variables. We calculate the velocity field $u$, starting with

$$
\Lambda^{\alpha-2} \rho=c_{n, \alpha} \int_{\mathbb{R}^{n}} \rho(\mathbf{x}-\mathbf{y}) \frac{1}{|\mathbf{y}|^{n+\alpha-2}} d \mathbf{y} .
$$

Then, we obtain $u$ by taking the gradient of the potential

$$
u_{i}(\mathbf{x})=\partial_{x_{i}} \Lambda^{\alpha-2} \rho=c_{n, \alpha} \int_{\mathbb{R}^{n}}\left(\rho\left(x_{1}-y_{1}\right)-\rho\left(x_{1}\right)\right) \frac{y_{i}}{|\mathbf{y}|^{n+\alpha}} d \mathbf{y}
$$

For $i=2, \cdots, n$, we have

$$
u_{i}(\mathbf{x})=c_{n, \alpha} \int_{\mathbb{R}}\left(\rho\left(x_{1}-y_{1}\right)-\rho\left(x_{1}\right)\right)\left[\int_{\mathbb{R}^{n-1}} \frac{y_{i}}{|\mathbf{y}|^{n+\alpha}} d y_{2} \cdots d y_{n}\right] d y_{1}=0 .
$$

The last eqaulity is due to oddness of the inside integral with respect to $y_{i}$.

For $i=1$,

$$
u_{1}(\mathbf{x})=c_{n, \alpha} \int_{\mathbb{R}}\left(\rho\left(x_{1}-y_{1}\right)-\rho\left(x_{1}\right)\right) y_{1}\left[\int_{\mathbb{R}^{n-1}} \frac{1}{|\mathbf{y}|^{n+\alpha}} d y_{2} \cdots d y_{n}\right] d y_{1} .
$$

Compute the integral inside,

$$
\begin{gathered}
\int_{\mathbb{R}^{n-1}} \frac{1}{|\mathbf{y}|^{n+\alpha}} d y_{2} \cdots d y_{n}=\int_{\mathbb{R}^{n-1}}\left(y_{1}^{2}+y_{2}^{2}+\cdots+y_{n}^{2}\right)^{-\frac{n+\alpha}{2}} d y_{2} \cdots d y_{n} \\
=\left|y_{1}\right|^{-(n+\alpha)} \int_{\mathbb{R}^{n-1}}\left(1+y_{2}^{2}+\cdots+y_{n}^{2}\right)^{-\frac{n+\alpha}{2}}\left|y_{1}\right|^{n-1} d y_{2} \cdots d y_{n} \\
=\left|y_{1}\right|^{-1-\alpha} \omega_{n-1} \int_{0}^{\infty}\left(1+r^{2}\right)^{-\frac{n+\alpha}{2}} r^{n-2} d r=c_{n, \alpha}^{\prime}\left|y_{1}\right|^{-1-\alpha} .
\end{gathered}
$$


Here, $\omega_{n}$ denotes the area of the unit sphere in $n$ dimension. The constant $c_{n, \alpha}^{\prime}$ is clearly positive, finite, and only depend on $n$ and $\alpha$.

Then, we obtain

$$
u_{1}(\mathbf{x})=c_{n, \alpha} c_{n, \alpha}^{\prime} \int_{\mathbb{R}} \frac{\rho\left(x_{1}-y_{1}\right)-\rho\left(x_{1}\right)}{\operatorname{sgn}\left(y_{1}\right)\left|y_{1}\right|^{\alpha}} d y_{1} .
$$

So, $u_{1}(\mathbf{x})=u_{1}\left(x_{1}\right)$ is also a constant in $\left(x_{2}, \cdots, x_{n}\right)$. Moreover, as a function of $x_{1}$, the expression of $u_{1}$ is the same as (15), except the constant $c_{\alpha}$ might be different.

From (27) and (28), we have

$$
\nabla \cdot(\rho(\mathbf{x}) \mathbf{u}(\mathbf{x}))=\partial_{x_{1}}\left(\rho\left(x_{1}\right) u_{1}\left(x_{1}\right)\right) .
$$

This implies if $\rho_{0}(\mathbf{x})=\rho_{0}\left(x_{1}\right)$, then $\rho(\mathbf{x}, t)=\rho\left(x_{1}, t\right)$. Moreover, $\left(\rho, u_{1}\right)$ as functions of $x_{1}$, will be the solution of the 1D system (11)-(3). Hence, Theorem 1.1 can be extended to multi-dimension, with the choice of initial data $\rho_{0}(\mathbf{x})=\rho_{0}\left(x_{1}\right)$, where $\rho_{0}$ as a function of $x_{1}$ is chosen the same way as in the 1D case. The different constant in (28) mentioned above will only affect the choice of $\delta$ throughout the proof.

We summarize the discussion to the following theorem.

Theorem 5.1. Consider the initial value problem of system (26) in the periodic domain $\mathbb{T}^{n}$. There exists a family of smooth initial data $\rho_{0}$ such that the solution loses uniform $C^{1}$ regularity.

5.2. Fractional Euler-Alignment system. The multi-dimensional Euler-Alignment system with singular influence function takes the form

$$
\partial_{t} \rho+\nabla \cdot(\rho \mathbf{u})=0, \quad \partial_{t} \mathbf{u}+\mathbf{u} \cdot \nabla \mathbf{u}=c_{n, \alpha} \int_{\mathbb{R}^{n}} \frac{\mathbf{u}(y, t)-\mathbf{u}(x, t)}{|y-x|^{n+\alpha}} \rho(y, t) d y .
$$

Let $G=\nabla \cdot \mathbf{u}-\Lambda^{\alpha} \rho$. Then, the dynamics of $G$ reads

$$
\partial_{t} G+\nabla \cdot(G \mathbf{u})=\operatorname{tr}\left(\nabla \mathbf{u}^{\otimes 2}\right)-(\nabla \cdot \mathbf{u})^{2} .
$$

Note that In the $1 \mathrm{D}$ case, the right hand side becomes $\left(\partial_{x} u\right)^{2}-\left(\partial_{x} u\right)^{2}=0$. Then, the dynamics becomes (12), and as a special case of $G \equiv 0$, we reach our system (1)-(2).

However, the right hand side is not necessarily zero in higher dimensions. This quantity is known as spectral gap. In particular, it destroys the maximum principle on $G$, and hence $G_{0} \equiv 0$ does not imply $G(\cdot, t) \equiv 0$.

Therefore, fractional porous median flow (26) is not a special case of the Euler-Alignment system, except in 1D. The global regularity on (29) for $\rho_{0}>0$ is an open problem. The main difficulty is the lack of apriori control of the spectral gap.

To construct $\rho_{0} \geq 0$ which leads to singularity formations, we can avoid the difficulty by select a special family of initial data such that the spectral gap is zero in all time.

The choices of $\left(\rho_{0}, \mathbf{u}_{0}\right)$ is the same as Section 5.1;

$$
\rho_{0}(\mathbf{x})=\rho_{0}\left(x_{1}\right), \quad\left(u_{0}\right)_{1}(\mathbf{x})=\left(u_{0}\right)_{1}\left(x_{1}\right), \quad\left(u_{0}\right)_{i}(\mathbf{x})=0, \forall i=2, \cdots, n .
$$

By the same argument, we know such structure preserves in time. So,

$$
\operatorname{tr}\left(\nabla \mathbf{u}^{\otimes 2}\right)-(\nabla \cdot \mathbf{u})^{2}=\left(\partial_{x_{1}} u_{1}\right)^{2}-\left(\partial_{x_{1}} u_{1}\right)^{2}=0 .
$$

Therefore, we pick $\rho_{0}$ the same as in Theorem [5.1, and $\mathbf{u}_{0}=\nabla \Lambda^{\alpha-2} \rho_{0}$. The solution will form singularities the same way as (26).

Corollary 5.2. Consider the initial value problem of system (29) in the periodic domain $\mathbb{T}^{n}$. There exists a family of smooth initial data $\left(\rho_{0}, \mathbf{u}_{0}\right)$ such that the solution loses uniform $C^{1}$ regularity. 


\section{FurTher Discussions}

Theorem 1.1 shows singularity formations for equations (11)-(3). However, it does not specify whether the blowup happens in finite time or when time approaches infinity.

For the special case with $\alpha=1$ and initial data (6), a finite time blowup was shown in [8]. Therefore, a reasonable conjecture would be, the singularity formations happen at a finite time.

The proof of the conjecture will require a stronger estimate on the velocity field

$$
u(x, t) \leq-C x^{\gamma},
$$

with $\gamma<1$. This will ensure the characteristic paths intersect in finite time, causing a blowup. To obtain the strong inequality, a delicate estimate to the singular integral near the singularity is required. We will leave it for future investigations.

Acknowledgments. This work is supported by NSF grant DMS 1853001. The author would like to thank Tam Do, Alexander Kiselev and Xiaoqian Xu for valuable discussions. The author also thank the referees for valubale suggestions.

\section{REFERENCES}

[1] Piotr Biler, Grzegorz Karch, and Régis Monneau. Nonlinear diffusion of dislocation density and selfsimilar solutions. Communications in Mathematical Physics, 294(1):145-168, 2010.

[2] Luis A Caffarelli, Fernando Soria, and Juan L Vázquez. Regularity of solutions of the fractional porous medium flow. Journal of the European Mathematical Society, 15(5):1701-1746, 2013.

[3] Luis A Caffarelli and Juan L Vázquez. Nonlinear porous medium flow with fractional potential pressure. Archive for Rational Mechanics and Analysis, 202(537-565), 2011.

[4] Luis A Caffarelli and Juan L Vázquez. Regularity of solutions of the fractional porous medium flow with exponent 1/2. St. Petersburg Mathematical Journal, 27(3):437-460, 2016.

[5] José A Carrillo, Young-Pil Choi, Eitan Tadmor, and Changhui Tan. Critical thresholds in 1D Euler equations with nonlocal forces. Mathematical Models and Methods in Applied Sciences, 26(1):185-206, 2016.

[6] José A Carrillo, Lucas CF Ferreira, and Juliana C Precioso. A mass-transportation approach to a one dimensional fluid mechanics model with nonlocal velocity. Advances in Mathematics, 231(1):306-327, 2012 .

[7] A Castro and D Córdoba. Global existence, singularities and ill-posedness for a nonlocal flux. Advances in Mathematics, 219(6):1916-1936, 2008.

[8] Dongho Chae, Antonio Córdoba, Diego Córdoba, and Marco A Fontelos. Finite time singularities in a 1D model of the quasi-geostrophic equation. Advances in Mathematics, 194(1):203-223, 2005.

[9] Antonio Córdoba, Diego Córdoba, and Marco A Fontelos. Formation of singularities for a transport equation with nonlocal velocity. Annals of mathematics, pages 1377-1389, 2005.

[10] Felipe Cucker and Steve Smale. Emergent behavior in flocks. Automatic Control, IEEE Transactions on, 52(5):852-862, 2007.

[11] Tam Do, Alexander Kiselev, Lenya Ryzhik, and Changhui Tan. Global regularity for the fractional Euler alignment system. Archive for Rational Mechanics and Analysis, 228(1):1-37, 2018.

[12] Seung-Yeal Ha and Eitan Tadmor. From particle to kinetic and hydrodynamic descriptions of flocking. Kinetic and Related Models, 1(3):415-435, 2008.

[13] Alexander Kiselev, Fedor Nazarov, and Roman Shterenberg. Blow up and regularity for fractal burgers equation. Dynamics of PDE, 5(3):211-240, 2008.

[14] Alexander Kiselev and Changhui Tan. Global regularity for 1D Eulerian dynamics with singular interaction forces. SIAM Journal on Mathematical Analysis, 50(6):6208-6229, 2018.

[15] Roman Shvydkoy and Eitan Tadmor. Eulerian dynamics with a commutator forcing. Transactions of Mathematics and Its Applications, 1(1), 2017.

[16] Roman Shvydkoy and Eitan Tadmor. Eulerian dynamics with a commutator forcing iii. fractional diffusion of order $0<\alpha<1$. Physica D: Nonlinear Phenomena, 2017.

[17] Luis Silvestre and Vlad Vicol. On a transport equation with nonlocal drift. Transactions of the American Mathematical Society, 368(9):6159-6188, 2016. 
[18] Eitan Tadmor and Changhui Tan. Critical thresholds in flocking hydrodynamics with non-local alignment. Philosophical Transactions of the Royal Society of London A: Mathematical, Physical and Engineering Sciences, 372(2028):20130401, 2014.

[19] Changhui Tan. On the euler-alignment system with weakly singular communication weights. arXiv preprint arXiv:1901.02582, 2019.

(Changhui Tan)

Department of Mathematics, University of South Carolina, 1523 Greene St., Columbia, SC 29208, USA

E-mail address: tan@math.sc.edu 